\title{
INTEGRATION BY PARTS FOR POINT PROCESSES AND MONTE CARLO ESTIMATION
}

\author{
NICOLAS PRIVAULT, ${ }^{*}$ Université de Poitiers \\ XIAO WEI, ${ }^{* *}$ Central University of Finance and Economics
}

\begin{abstract}
We develop an integration by parts technique for point processes, with application to the computation of sensitivities via Monte Carlo simulations in stochastic models with jumps. The method is applied to density estimation with respect to the Lebesgue measure via a modified kernel estimator which is less sensitive to variations of the bandwidth parameter than standard kernel estimators. This applies to random variables whose densities are not analytically known and requires the knowledge of the point process jump times.
\end{abstract}

Keywords: Malliavin calculus; point process; renewal process; sensitivity analysis; density estimation; kernel estimator

2000 Mathematics Subject Classification: Primary 62G07

Secondary 60H07; 65C05; 60G55; 60K15

\section{Introduction}

Kernel estimators for the density $\phi_{F}$ of a random variable $F$ from a random sample $\{F(k)\}_{k=1, \ldots, N}$ of $F$ have been introduced in [17] and [14]. More precisely in [17], finite difference estimators of the form

$$
\phi_{F}(y) \simeq \frac{1}{h} \mathrm{E}\left[\mathbf{1}_{[-h / 2, h / 2]}(F-y)\right] \simeq \frac{1}{2 N h} \sum_{k=1}^{N} \mathbf{1}_{[-h, h]}(F(k)-y), \quad y \in \mathbb{R}_{+},
$$

have been constructed and extended in [14] to estimators of the form

$$
\phi_{F}(y) \simeq \frac{1}{N h} \sum_{k=1}^{N} K\left(\frac{F(k)-y}{h}\right)
$$

where $K: \mathbb{R} \rightarrow \mathbb{R}_{+}$is a kernel satisfying

$$
\int_{-\infty}^{\infty} K(x) \mathrm{d} x=1
$$

The performance of kernel estimators is dependent on the choice of the bandwidth parameter $h$, whose optimal value is a function of the number of samples, $N$, i.e. it should decrease as $N$ increases. It has been known since [17] that the optimal rate of decrease of $h$ in the mean

Received 3 February 2005; revision received 22 May 2007.

* Current address: Department of Mathematics, City University of Hong Kong, Tat Chee Avenue, Kowloon, Hong Kong. Email address: nprivaul@cityu.edu.hk

** Postal address: School of Insurance, Central University of Finance and Economics, Beijing, 100081, P. R. China.

Email address: weix@cufe-ins.sina.net 
square sense is $N^{-1 / 4}$ for the finite difference estimator, while in [14] optimal values of $h$ have been obtained for kernel estimators in terms of $N$ and $K$.

Conversely, integration by parts and related Malliavin calculus techniques can be used to represent the density $\phi_{F}$ of $F$ with respect to the Lebesgue measure as

$$
\phi_{F}(y)=\frac{\partial}{\partial y} \mathrm{P}(F \leq y)=\mathrm{E}\left[W \mathbf{1}_{\{F \leq y\}}\right],
$$

under certain technical assumptions; see, e.g. Section 2.1 of [13] on the Wiener space, where $W$ is a random variable called a weight. This provides another way to estimate the density of $F$ with respect to the Lebesgue measure using Monte Carlo methods: denoting by $\{F(k)\}_{k=1, \ldots, N}$ a random sample distributed according to the law of $F$ we have

$$
\phi_{F}(y) \simeq \frac{1}{N} \sum_{k=1}^{N} W(k) \mathbf{1}_{\{F(k) \leq y\}},
$$

where $\{W(k)\}_{k=1, \ldots, N}$ denotes independent random samples of $W$. The interest in (1.3), compared to kernel estimators, is that it is independent of the value of a bandwidth parameter. Note, however, that in addition to the samples of $F$, this estimator requires the knowledge of the random path of the underlying stochastic process in order to evaluate $W$. Conversely, the integrability of the weight $W$ in (1.2) entails the existence of the density of $F$ with respect to the Lebesgue measure, thus excluding discrete random variables from this approach.

More generally, the Malliavin calculus has been applied to sensitivity analysis in continuous and discontinuous financial markets (see [1], [2], [6], [7], [8], [10], and [11]) and in insurance (see [16]), to express derivatives of the form $\partial \mathrm{E}\left[f\left(F_{\zeta}\right)\right] / \partial \zeta$ as

$$
\frac{\partial}{\partial \zeta} \mathrm{E}\left[f\left(F_{\zeta}\right)\right]=\mathrm{E}\left[W_{\zeta} f\left(F_{\zeta}\right)\right],
$$

where $\left(F_{\zeta}\right)$ is a family of random variables in $\varsigma_{T}$ depending on a parameter $\zeta \in \mathbb{R}$. Here, $W_{\zeta}$ is a weight independent of the function $f$, which need not be differentiable; in particular, the estimation of the density, (1.3), corresponds to $f=\mathbf{1}_{(-\infty, 0)}$ and $F_{y}=F-y$, with $W$ independent of $y$. Note that in mathematical finance, each value of the bandwidth parameter $h$ in the finite difference

$$
\frac{1}{2 h} \mathrm{E}\left[f\left(F_{\zeta+h}\right)-f\left(F_{\zeta-h}\right)\right]
$$

yields a different estimate of the corresponding sensitivity (also called 'Greek'), see, e.g. [5, p. 40], whereas (1.4) is again independent of a bandwidth parameter.

In Proposition 3.1, below, we derive a general integration by parts formula for point processes, extending the results obtained in the Poisson case in [3], [8], [9], [15], and [16], with potential application to sensitivity analysis and density estimation for stochastic models in finance, insurance, and engineering. Using this integration by parts formula we obtain an expression of the form (1.2)-(1.3) for the density of a random functional $F$ with respect to the Lebesgue measure:

$$
\phi_{F}(y)=\mathrm{E}\left[W \mathbf{1}_{\{F \leq y\}}\right] \simeq \frac{1}{N} \sum_{k=1}^{N} W(k) \mathbf{1}_{\{F(k) \leq y\}} .
$$

This expression requires the knowledge of the characteristics (the Janossy densities) of the underlying point process in order to compute the weight $W$, while the density of $F$ may be unknown or not analytically computable and thus requires estimating numerically. 
It turns out that the performance of the corresponding estimator (1.5) decreases when $y$ is large, in which case the term $W \mathbf{1}_{\{F \leq y\}}$ has a large variance. This problem is tackled by a localization procedure, which involves mixing (1.5) with a standard kernel estimate, i.e.

$$
\begin{aligned}
\phi_{F}(y) & =\frac{1}{\eta} \mathrm{E}\left[K\left(\frac{F-y}{\eta}\right)\right]-\mathrm{E}\left[W f\left(\frac{F-y}{\eta}\right)\right] \\
& \simeq \frac{1}{N \eta} \sum_{k=1}^{N} K\left(\frac{F(k)-y}{\eta}\right)-\frac{1}{N} \sum_{k=1}^{N} W(k) f\left(\frac{F(k)-y}{\eta}\right),
\end{aligned}
$$

where $K$ is a kernel supported in $[0, \infty)$ and

$$
f(x)=\mathbf{1}_{[0, \infty)}(x)\left(1-\int_{0}^{x} K(y) \mathrm{d} y\right), \quad x \in \mathbb{R} .
$$

As shown in Section 6, this estimator combines the advantages of Malliavin-type estimators, (1.5), and kernel estimators, (1.1), in that it is little sensitive to values of the bandwidth parameter $h$, while at the same time it does not present the above mentioned variance problem. Relation (1.6) can be viewed as the point process analog of a result proved in [12] on the Wiener space, see Theorem 2.1 therein. The optimization results of [12], in terms of the kernel $K$ and the bandwidth parameter $h$, also apply here and are used in the numerical simulations; see Section 6.3.

We proceed as follows. In Section 2 we review some properties of point processes, and in Section 3 we establish the integration by parts formula (Proposition 3.1), which will be our main tool for density estimation. In Section 4 we present an application of the integration by parts formula to the computation of sensitivities, in particular, for functionals of the form

$$
F=\int_{0}^{T} h(t) \mathrm{d} X_{t}
$$

where $h$ is a $\mathcal{C}^{1}$ function and

$$
X_{t}=\sum_{k=1}^{N_{t}} Y_{k}, \quad t \in \mathbb{R}_{+},
$$

is a compound log-normal renewal process with random marks $\left(Y_{k}\right)_{k \geq 1}$ independent of $\left(N_{t}\right)_{t \in \mathbb{R}_{+}}$. These results are used in Section 5 to construct a modified kernel density estimator. Simulations and comparisons of different methods for density estimation are presented in Section 6 for functionals of the form of (1.7) with $h(t)=\mathrm{e}^{-r t}, t \in[0, T]$. Such functionals can be used to express risk reserve processes for insurance portfolios in which the accumulated amount of claims occurring in the time interval $(0, t]$ is given by $X_{t}$; see, e.g. [16].

\section{Point processes}

Let

$$
N_{t}=\sum_{k=1}^{\infty} \mathbf{1}_{\left[T_{k}, \infty\right)}(t), \quad t \in \mathbb{R}_{+},
$$

be a point process with an increasing sequence of jump times $\left(T_{k}\right)_{k \geq 1}$, generating the filtration $\left(\mathcal{F}_{t}\right)_{t \in \mathbb{R}_{+}}$on a probability space $(\Omega, \mathcal{F}, \mathrm{P})$. Set $T_{0}=0$ and let the interjump times of $\left(N_{t}\right)_{t \in \mathbb{R}_{+}}$ be denoted by $\tau_{k}:=T_{k}-T_{k-1}, k \geq 1$. 
Definition 2.1. Let $T>0$. We denote by $\varsigma_{T}$ the subspace of $L^{2}\left(\Omega, \mathcal{F}_{T}\right)$ made of functionals of the form

$$
F=f_{0} \mathbf{1}_{\left\{N_{T}=0\right\}}+\sum_{n=1}^{\infty} \mathbf{1}_{\left\{N_{T}=n\right\}} f_{n}\left(T_{1}, \ldots, T_{n}\right),
$$

where $f_{0} \in \mathbb{R}$ and $f_{n}$ is $\mathcal{C}^{2}$ and symmetric in $n$ variables on $[0, T]^{n}, n \geq 1, T>0$.

The set of $F \in \wp_{T}$ for which (2.2) is finite is denoted by $\delta_{T}^{f}$ and is dense in $L^{p}\left(\Omega, \mathcal{F}_{T}\right)$, $p \geq 1$. The expectation of $F$ equals

$$
\mathrm{E}[F]=j_{T, 0} f_{0}+\sum_{n=1}^{\infty} \frac{1}{n !} \int_{0}^{T} \cdots \int_{0}^{T} f_{n}\left(t_{1}, \ldots, t_{n}\right) j_{T, n}\left(t_{1}, \ldots, t_{n}\right) \mathrm{d} t_{1} \cdots \mathrm{d} t_{n},
$$

where $j_{T, n}: \mathbb{R}_{+}^{n} \rightarrow \mathbb{R}_{+}, n \geq 1$, are nonnegative symmetric functions on $[0, T]^{n}$ called the Janossy densities and $j_{T, 0} \in \mathbb{R}_{+}$; see [18], [4, Section 5.3], and the references therein. In other terms, we have

$$
\mathrm{P}\left(T_{1} \in \mathrm{d} t_{1}, \ldots, T_{n} \in \mathrm{d} t_{n}, N_{T}=n\right)=j_{T, n}\left(t_{1}, \ldots, t_{n}\right) \mathrm{d} t_{1} \cdots \mathrm{d} t_{n},
$$

$0 \leq t_{1}<t_{2}<\cdots<t_{n} \leq T$. We turn to some examples of point processes and their Janossy densities.

Example 2.1. (Poisson processes.) In the case of Poisson processes with arbitrary deterministic intensity $\lambda(t)$ we have

$$
j_{T, n}\left(t_{1}, \ldots, t_{n}\right)=\lambda\left(t_{1}\right) \cdots \lambda\left(t_{n}\right) \exp \left(-\int_{0}^{T} \lambda(t) \mathrm{d} t\right),
$$

i.e. for the standard Poisson process with intensity $\lambda>0$ we have

$$
j_{T, n}\left(t_{1}, \ldots, t_{n}\right)=\lambda^{n} \mathrm{e}^{-\lambda T}, \quad t_{1}, \ldots, t_{n} \in[0, T] .
$$

Example 2.2. (Renewal processes.) A point process $\left(N_{t}\right)_{t \in \mathbb{R}_{+}}$as defined in (2.1) is called a renewal process with interoccurrence time distribution function $Z(x)$ and density $z(x)$ if the random variables $\tau_{k}=T_{k}-T_{k-1}, k \geq 1$, are independent and identically distributed (i.i.d.) with

$$
Z(x)=\mathrm{P}\left(\tau_{k} \leq x\right)=\int_{0}^{x} z(y) \mathrm{d} y, \quad x \in \mathbb{R}_{+}, k \geq 1 .
$$

Since the sequence $\left(\tau_{k}\right)_{k \geq 1}$ is i.i.d., for $0 \leq t_{1}<t_{2}<\cdots<t_{n} \leq T$, we have

$$
\begin{aligned}
\mathrm{P}\left(T_{1}\right. & \left.\in \mathrm{d} t_{1}, \ldots, T_{n} \in \mathrm{d} t_{n}, N_{T}=n\right) \\
& =\mathrm{P}\left(\tau_{1} \in \mathrm{d} t_{1}, t_{1}+\tau_{2} \in \mathrm{d} t_{2}, \ldots, t_{n-1}+\tau_{n} \in \mathrm{d} t_{n}, \tau_{n+1}>T-t_{n}\right) \\
& =z\left(t_{1}\right) z\left(t_{2}-t_{1}\right) \cdots z\left(t_{n}-t_{n-1}\right)\left(1-Z\left(T-t_{n}\right)\right) \mathrm{d} t_{1} \cdots \mathrm{d} t_{n} ;
\end{aligned}
$$

hence, the Janossy densities $j_{T, n}\left(t_{1}, \ldots, t_{n}\right)$ are given by

$$
j_{T, n}\left(t_{1}, \ldots, t_{n}\right)=z\left(t_{1}\right) z\left(t_{2}-t_{1}\right) \cdots z\left(t_{n}-t_{n-1}\right) \int_{T-t_{n}}^{\infty} z(s) \mathrm{d} s
$$

for $0 \leq t_{1}<\cdots<t_{n} \leq T$. The value of $j_{T, n}\left(t_{1}, \ldots, t_{n}\right)$ on $\left(t_{1}, \ldots, t_{n}\right) \in[0, T]^{n}$ is obtained by symmetrization, i.e.

$$
j_{T, n}\left(t_{1}, \ldots, t_{n}\right)=j_{T, n}\left(t_{(1)}, \ldots, t_{(n)}\right), \quad t_{1}, \ldots, t_{n} \in[0, T],
$$

where $\left(t_{(1)}, \ldots, t_{(n)}\right)$ denotes the sequence $\left(t_{1}, \ldots, t_{n}\right)$ in ascending order; see [4, Section 5.3]. 


\section{Integration by parts}

Definition 3.1. Given $w \in \mathcal{C}^{1}([0, T])$, let $D_{w}$ denote the gradient operator defined on $F \in \varsigma_{T}$, of the form of (2.2), and given by

$$
D_{w} F=-\sum_{n=1}^{\infty} \mathbf{1}_{\left\{N_{T}=n\right\}} \sum_{k=1}^{n} w\left(T_{k}\right) \frac{\partial f_{n}}{\partial t_{k}}\left(T_{1}, \ldots, T_{n}\right) .
$$

Let $\mathcal{C}_{0}^{1}([0, T])$ denote the space of $w \in \mathcal{C}^{1}([0, T])$ such that $w(0)=w(T)=0$. In the sequel we assume that $j_{T, n} \in \mathcal{C}^{1}\left([0, T]^{n}\right), n \geq 1$. Next, we state the definition of the divergence operator.

Definition 3.2. Given $w \in \mathcal{C}_{0}^{1}([0, T])$ and $G \in \varsigma_{T}$, let

$$
D_{w}^{*} G=G \int_{0}^{T} w^{\prime}(t) \mathrm{d} N_{t}-G D_{w} \log \left|G j_{T, N_{T}}\left(T_{1}, \ldots, T_{N_{T}}\right)\right|,
$$

with the convention that $0 / 0=0$.

Fix $p, q>1$ satisfying $1 / p+1 / q=1$ and let $\operatorname{Dom}_{p}\left(D_{w}\right)$ and $\operatorname{Dom}_{q}\left(D_{w}^{*}\right)$ be respectively defined as the sets of functionals $F \in L^{p}\left(\Omega, \mathcal{F}_{T}\right)$ and $F \in L^{q}\left(\Omega, \mathcal{F}_{T}\right)$, for which there exists $\left(F_{n}\right)_{n \in N}$ in $\delta_{T}^{f}$ converging to $F$ in $L^{p}\left(\Omega, \mathcal{F}_{T}\right)$ and $L^{q}\left(\Omega, \mathcal{F}_{T}\right)$, respectively, and such that $\left(D_{w} F_{n}\right)_{n \in N}$ and $\left(D_{w}^{*} F_{n}\right)_{n \in N}$ respectively converge in $L^{p}\left(\Omega, \mathcal{F}_{T}\right)$ and $L^{q}\left(\Omega, \mathcal{F}_{T}\right)$. In the next proposition we extend the integration by parts formulas of [3] and [16] to the setting of point processes.

Proposition 3.1. Let $w \in \mathcal{C}_{0}^{1}([0, T])$. The operators $D_{w}$ and $D_{w}^{*}$ are closable and can be extended to their closed domains $\operatorname{Dom}_{p}\left(D_{w}\right)$ and $\operatorname{Dom}_{q}\left(D_{w}^{*}\right)$ with the duality relation

$$
\mathrm{E}\left[G D_{w} F\right]=\mathrm{E}\left[F D_{w}^{*} G\right], \quad F \in \operatorname{Dom}_{p}\left(D_{w}\right), G \in \operatorname{Dom}_{q}\left(D_{w}^{*}\right) .
$$

Proof. For any $F \in s_{T}^{f}$, we have

$$
\begin{aligned}
\mathrm{E}\left[D_{w} F\right] & =-\sum_{n=1}^{\infty} \frac{1}{n !} \int_{0}^{T} \cdots \int_{0}^{T} \sum_{k=1}^{n} w\left(t_{k}\right) \frac{\partial f_{n}}{\partial t_{k}}\left(t_{1}, \ldots, t_{n}\right) j_{T, n}\left(t_{1}, \ldots, t_{n}\right) \mathrm{d} t_{1} \cdots \mathrm{d} t_{n} \\
& =\sum_{n=1}^{\infty} \frac{1}{n !} \int_{0}^{T} \cdots \int_{0}^{T} f_{n}\left(t_{1}, \ldots, t_{n}\right) \sum_{k=1}^{n} \frac{\partial}{\partial t_{k}}\left(w\left(t_{k}\right) j_{T, n}\left(t_{1}, \ldots, t_{n}\right)\right) \mathrm{d} t_{1} \cdots \mathrm{d} t_{n} \\
& =\sum_{n=1}^{\infty} \frac{1}{n !} \int_{0}^{T} \cdots \int_{0}^{T} f_{n}\left(t_{1}, \ldots, t_{n}\right) j_{T, n}\left(t_{1}, \ldots, t_{n}\right) \\
& \times\left(\sum_{k=1}^{n} w^{\prime}\left(t_{k}\right)+\sum_{k=1}^{n} w\left(t_{k}\right) \frac{\partial \log j_{T, n}}{\partial t_{k}}\left(t_{1}, \ldots, t_{n}\right)\right) \mathrm{d} t_{1} \cdots \mathrm{d} t_{n} \\
& =\mathrm{E}\left[\left(\int_{0}^{T} w^{\prime}(t) \mathrm{d} N_{t}-D_{w} \log j_{T, N_{T}}\left(T_{1}, \ldots, T_{N_{T}}\right)\right) F\right] ;
\end{aligned}
$$


hence, for all $F, G \in s_{T}^{f}$, we obtain

$$
\begin{aligned}
\mathrm{E}\left[G D_{w} F\right] & =\mathrm{E}\left[D_{w}(F G)-F D_{w} G\right] \\
& =\mathrm{E}\left[F\left(G \int_{0}^{T} w^{\prime}(t) \mathrm{d} N_{t}-G D_{w} \log j_{T, N_{T}}\left(T_{1}, \ldots, T_{N_{T}}\right)-D_{w} G\right)\right] \\
& =\mathrm{E}\left[F D_{w}^{*} G\right] .
\end{aligned}
$$

Now, let $\left(F_{n}\right)_{n \in N}$ and $\left(\tilde{F}_{n}\right)_{n \in N}$ be two sequences in $s_{T}^{f}$ converging to the same $F$ in $L^{p}\left(\Omega, \tilde{F}_{T}\right)$ such that both $\left(D_{w} F_{n}\right)_{n \in N}$ and $\left(D_{w} \tilde{F}_{n}\right)_{n \in N}$ have limits denoted by $U$ and $V$ in $L^{p}\left(\Omega, \mathcal{F}_{T}\right)$. For all $G \in s_{T}^{f}$, we have

$$
\begin{aligned}
\left|\langle U-V, G\rangle_{L^{2}}\right| & =\lim _{n \rightarrow \infty}\left|\left\langle D_{w} F_{n}-D_{w} \tilde{F}_{n}, G\right\rangle_{L^{2}}\right| \\
& =\left|\lim _{n \rightarrow \infty}\left\langle F_{n}-\tilde{F}_{n}, D_{w}^{*} G\right\rangle_{L^{2}}\right| \\
& \leq\left\|D_{w}^{*} G\right\|_{L^{q}} \lim _{n \rightarrow \infty}\left\|F_{n}-\tilde{F}_{n}\right\|_{L^{p}} \\
& =0 ;
\end{aligned}
$$

hence, $U=V$, P-almost surely. This shows that $D_{w}$ can be extended to $F \in \operatorname{Dom}_{p}\left(D_{w}\right)$ by letting

$$
D_{w} F=\lim _{n \rightarrow \infty} D_{w} F_{n}
$$

for any sequence $\left(F_{n}\right)_{n \in N} \subset \operatorname{Dom}_{p}\left(D_{w}\right)$ converging to $F$ in $L^{p}\left(\Omega, \mathcal{F}_{T}\right)$, and such that $\left(D_{w} F_{n}\right)_{n \in N}$ converges in $L^{p}\left(\Omega, \mathcal{F}_{T}\right)$. A similar argument applies to $D_{w}^{*}$ and allows us to extend the duality relation (3.2) to all $F \in \operatorname{Dom}_{p}\left(D_{w}\right)$ and $G \in \operatorname{Dom}_{q}\left(D_{w}^{*}\right)$.

We note the following.

Assertion 3.1. Let $F \in \varsigma_{T}$ such that $F \in L^{p}\left(\Omega, \mathcal{F}_{T}\right)$, respectively $F \in L^{q}\left(\Omega, \mathcal{F}_{T}\right)$, and $D_{w} F \in L^{p}\left(\Omega, \mathcal{F}_{T}\right)$, respectively $D_{w}^{*} F \in L^{q}\left(\Omega, \widetilde{F}_{T}\right)$.

Proof. It suffices to approximate $F$, written as in (2.2), by the truncated sequence

$$
F_{m}=f_{0} \mathbf{1}_{\left\{N_{T}=0\right\}}+\sum_{n=1}^{m} \mathbf{1}_{\left\{N_{T}=n\right\}} f_{n}\left(T_{1}, \ldots, T_{n}\right), \quad m \geq 1,
$$

and to note that $\left(D_{w} F_{m}\right)_{m \geq 1}$ and $\left(D_{w}^{*} F_{m}\right)_{m \geq 1}$ are convergent in $L^{p}\left(\Omega, \mathcal{F}_{T}\right)$ and $L^{q}\left(\Omega, \mathcal{F}_{T}\right)$, respectively.

This remark allows us to prove the following lemma, whose hypotheses will apply in the sequel.

Lemma 3.1. Let $p \geq 1$ and assume that there exists a $c_{0}>0$ such that

$$
j_{T, n}^{1-p}\left(t_{1}, \ldots, t_{n}\right)\left|\frac{\partial j_{T, n}}{\partial t_{k}}\left(t_{1}, \ldots, t_{n}\right)\right| \leq c_{0}^{n},
$$

$k=1, \ldots, n, t_{1}, \ldots, t_{n} \in[0, T]^{n}, n \geq 1$. Then $D_{w} \log j_{T, N_{T}}\left(T_{1}, \ldots, T_{N_{T}}\right) \in L^{p}\left(\Omega, \mathcal{F}_{T}\right)$. 
Proof. From (2.3) we have

$$
\begin{aligned}
& \left\|D_{w} \log j_{T, N_{T}}\left(T_{1}, \ldots, T_{N_{T}}\right)\right\|_{L^{p}}^{p} \\
& \quad=\sum_{n=1}^{\infty} \frac{1}{n !} \int_{0}^{T} \cdots \int_{0}^{T}\left|\sum_{k=1}^{n} w\left(t_{k}\right) \frac{\partial j_{T, n}}{\partial t_{k}}\left(t_{1}, \ldots, t_{n}\right)\right|^{p}\left|j_{T, n}\left(t_{1}, \ldots, t_{n}\right)\right|^{1-p} \mathrm{~d} t_{1} \cdots \mathrm{d} t_{n} \\
& \quad \leq\|w\|_{\infty}^{p} c_{0} T \mathrm{e}^{c_{0} T}
\end{aligned}
$$

hence,

$$
D_{w} \log j_{T, N_{T}}\left(T_{1}, \ldots, T_{N_{T}}\right) \in L^{p}\left(\Omega, \mathcal{F}_{T}\right)
$$

and $\log j_{T, N_{T}}\left(T_{1}, \ldots, T_{N_{T}}\right) \in L^{p}\left(\Omega, \mathcal{F}_{T}\right)$ follows in the same way.

We now turn to the calculation of $D_{w} \log j_{T, N_{T}}\left(T_{1}, \ldots, T_{N_{T}}\right)$ for examples of point processes satisfying (3.3) for all $p \geq 1$.

Example 3.1. (Poisson processes.) In the case of a Poisson process with arbitrary deterministic intensity $\lambda \in \mathcal{C}_{b}^{1}\left(\mathbb{R}_{+}\right)$we have

$$
\log j_{T, N_{T}}\left(T_{1}, \ldots, T_{N_{T}}\right)=\int_{0}^{T} \log \lambda(t) \mathrm{d} N_{t}-\int_{0}^{T} \lambda(t) \mathrm{d} t
$$

and

$$
D_{w} \log j_{T, N_{T}}\left(T_{1}, \ldots, T_{N_{T}}\right)=-\int_{0}^{T} w(t) \frac{\lambda^{\prime}(t)}{\lambda(t)} \mathrm{d} N_{t} .
$$

Example 3.2. (Renewal processes.) In this case, (2.4) yields

$$
\begin{aligned}
& D_{w} \log j_{T, N_{T}}\left(T_{1}, \ldots, T_{N_{T}}\right) \\
&=-\frac{w\left(T_{N_{T}}\right) z\left(T-T_{N_{T}}\right)}{1-Z\left(T-T_{N_{T}}\right)}-\sum_{k=1}^{N_{T}} w\left(T_{k}\right) \frac{z^{\prime}\left(T_{k}-T_{k-1}\right)}{z\left(T_{k}-T_{k-1}\right)}+\sum_{k=1}^{N_{T}-1} w\left(T_{k}\right) \frac{z^{\prime}\left(T_{k+1}-T_{k}\right)}{z\left(T_{k+1}-T_{k}\right)} \\
&=\int_{0}^{T} w(t)\left(\frac{z^{\prime}\left(T_{N_{t}+1}-T_{N_{t}}\right)}{z\left(T_{N_{t}+1}-T_{N_{t}}\right)}-\frac{z^{\prime}\left(T_{N_{t}}-T_{N_{t}-1}\right)}{z\left(T_{N_{t}}-T_{N_{t}-1}\right)}\right) \mathrm{d} N_{t} \\
&-w\left(T_{N_{T}}\right) \frac{z^{\prime}\left(T_{N_{T}+1}-T_{N_{T}}\right)}{z\left(T_{N_{T}+1}-T_{N_{T}}\right)}-\frac{w\left(T_{N_{T}}\right) z\left(T-T_{N_{T}}\right)}{1-Z\left(T-T_{N_{T}}\right)} \\
&= \int_{0}^{T}\left(w\left(t-\tau_{N_{t}}\right)-w(t)\right) \frac{z^{\prime}\left(T_{N_{t}}-T_{N_{t}-1}\right)}{z\left(T_{N_{t}}-T_{N_{t}-1}\right)} \mathrm{d} N_{t}-\frac{w\left(T_{N_{T}}\right) z\left(T-T_{N_{T}}\right)}{1-Z\left(T-T_{N_{T}}\right)} .
\end{aligned}
$$

Example 3.3. (Log-normal renewal process.) In this example the interarrival times are i.i.d. according to the log-normal distribution with parameter $\sigma>0$, i.e.

$$
z(x)=\frac{\exp \left(-(\log x)^{2} / 2 \sigma^{2}\right)}{\sigma x \sqrt{2 \pi}}, \quad x>0 .
$$


In other terms $T_{k}-T_{k-1}=\mathrm{e}^{\sigma \xi_{k}}$, where $\left(\xi_{k}\right)_{k \geq 1}$ is an i.i.d. sequence of standard Gaussian random variables, and

$$
\begin{aligned}
D_{w} \log & j_{T, N_{T}}\left(T_{1}, \ldots, T_{N_{T}}\right) \\
= & \sum_{k=1}^{N_{T}} \frac{w\left(T_{k}\right)}{T_{k}-T_{k-1}}\left(1+\frac{\log \left(T_{k}-T_{k-1}\right)}{\sigma^{2}}\right)-\sum_{k=1}^{N_{T}-1} \frac{w\left(T_{k}\right)}{T_{k+1}-T_{k}}\left(1+\frac{\log \left(T_{k+1}-T_{k}\right)}{\sigma^{2}}\right) \\
& -\frac{w\left(T_{N_{T}}\right) \exp \left(-\left(\log \left(T-T_{N_{T}}\right)\right)^{2} / 2 \sigma^{2}\right)}{\sigma \sqrt{2 \pi}\left(T-T_{N_{T}}\right)\left(1-Z\left(T-T_{N_{T}}\right)\right)} \\
= & \sum_{k=1}^{N_{T}} \frac{w\left(T_{k}\right)}{T_{k}-T_{k-1}}\left(1+\sigma^{-1} \xi_{k}\right)-\sum_{k=1}^{N_{T}-1} \frac{w\left(T_{k}\right)}{T_{k+1}-T_{k}}\left(1+\sigma^{-1} \xi_{k+1}\right) \\
& -\frac{w\left(T_{N_{T}}\right) \exp \left(-\left(\log \left(T-T_{N_{T}}\right)\right)^{2} / 2 \sigma^{2}\right)}{\sigma \sqrt{2 \pi}\left(T-T_{N_{T}}\right)\left(1-Z\left(T-T_{N_{T}}\right)\right)} \\
= & -\frac{w\left(T_{N_{T}}\right) \exp \left(-\left(\log \left(T-T_{N_{T}}\right)\right)^{2} / 2 \sigma^{2}\right)}{\sigma \sqrt{2 \pi}\left(T-T_{N_{T}}\right)\left(1-Z\left(T-T_{N_{T}}\right)\right)}+\sum_{k=1}^{N_{T}}\left(w\left(T_{k}\right)-w\left(T_{k-1}\right)\right) \frac{1+\sigma^{-1} \xi_{k}}{T_{k}-T_{k-1}} \\
= & -\frac{w\left(T_{N_{T}}\right) \exp \left(-\left(\log \left(T-T_{N_{T}}\right)\right)^{2} / 2 \sigma^{2}\right)}{\sigma \sqrt{2 \pi}\left(T-T_{N_{T}}\right)\left(1-Z\left(T-T_{N_{T}}\right)\right)}+\int_{0}^{T_{N_{T}}} w^{\prime}(s) \frac{1+\sigma^{-1} \xi_{1+N_{s}}}{\tau_{1+N_{s}}} \mathrm{~d} s .
\end{aligned}
$$

In the simulations of Section 5 we will take $w(t)=t(T-t), t \in[0, T]$. In this case we have

$$
\begin{aligned}
\int_{0}^{T} w^{\prime}(t) \mathrm{d} N_{t}-D_{w} \log j_{T, N_{T}}\left(T_{1}, \ldots, T_{N_{T}}\right) & \\
= & \frac{T_{N_{T}} \exp \left(-\left(\log \left(T-T_{N_{T}}\right)\right)^{2} / 2 \sigma^{2}\right)}{\left(1-Z\left(T-T_{N_{T}}\right)\right) \sigma \sqrt{2 \pi}}+\sum_{k=1}^{N_{T}}\left(T-2 T_{k}\right) \\
& -\sum_{k=1}^{N_{T}}\left(T-T_{k}-T_{k-1}\right)\left(1+\sigma^{-1} \xi_{k}\right) \\
= & \left(\frac{\exp \left(-\left(\log \left(T-T_{N_{T}}\right)\right)^{2} / 2 \sigma^{2}\right)}{\left(1-Z\left(T-T_{N_{T}}\right)\right) \sigma \sqrt{2 \pi}}-1\right) T_{N_{T}}-\sigma^{-1} \sum_{k=1}^{N_{T}}\left(T-T_{k}-T_{k-1}\right) \xi_{k} .
\end{aligned}
$$

\section{Sensitivity analysis}

Let $I=(a, b)$ be an open interval of $\mathbb{R}$ and consider the derivative

$$
\frac{\partial}{\partial \zeta} \mathrm{E}\left[f\left(F_{\zeta}\right)\right]=\mathrm{E}\left[\frac{\partial F_{\zeta}}{\partial \zeta} f^{\prime}\left(F_{\zeta}\right)\right], \quad \zeta \in(a, b),
$$

where $\left(F_{\zeta}\right)_{\zeta \in(a, b)}$ is a family of random variables differentiable in a parameter $\zeta$ and $f$ is a $\mathcal{C}^{1}$ function on $\mathbb{R}$. This expression can be approximated by finite differences as

$$
\frac{1}{2 h} \mathrm{E}\left[f\left(F_{\zeta+h}\right)-f\left(F_{\zeta-h}\right)\right]
$$

while (4.1) fails when $f$ is not differentiable, e.g. when $f=\mathbf{1}_{[0, \infty)}$. 
Proposition 4.1, below, provides an expression for this derivative without using finite differences or requiring the differentiability of $f$. This formula will be applied in Section 5 to numerical simulations that will be compared to the results given by kernel estimates.

In the sequel and in Propositions 4.1, 4.2, and 4.3 we consider a family $\left(F_{\zeta}\right)_{\zeta \in(a, b)}$ of random functionals, continuously differentiable in $\operatorname{Dom}_{p}\left(D_{w}\right)$ in the parameter $\zeta \in(a, b)$, such that, for some $n_{0} \in \mathbb{N}$,

$$
D_{w} F_{\zeta} \neq 0 \text { a.s. on }\left\{N_{T} \geq n_{0}\right\},
$$

where $w$ is a given element of $\mathcal{C}_{0}^{1}([0, T])$ and the function $f: \mathbb{R} \rightarrow \mathbb{R}$ is assumed to satisfy $f\left(F_{\zeta}\right) \in L^{p}\left(\Omega, \widetilde{F}_{T}\right)$ for all $\zeta \in(a, b)$.

Proposition 4.1. Assume that

$$
\mathbf{1}_{\left\{N_{T} \geq n_{0}\right\}} \frac{\partial_{\zeta} F_{\zeta}}{D_{w} F_{\zeta}} \in \operatorname{Dom}_{q}\left(D_{w}^{*}\right), \quad \zeta \in(a, b) .
$$

Then we have

$$
\frac{\partial}{\partial \zeta} \mathrm{E}\left[f\left(F_{\zeta}\right) \mid N_{T} \geq n_{0}\right]=\mathrm{E}\left[W_{\zeta} f\left(F_{\zeta}\right) \mid N_{T} \geq n_{0}\right], \quad \zeta \in(a, b),
$$

where the weight $W_{\zeta}$ is given by

$$
W_{\zeta}=D_{w}^{*}\left(\mathbf{1}_{\left\{N_{T} \geq n_{0}\right\}} \frac{\partial_{\zeta} F_{\zeta}}{D_{w} F_{\zeta}}\right), \quad \zeta \in(a, b) .
$$

Proof. Assuming that $f \in \mathcal{C}_{b}^{\infty}(\mathbb{R})$ we have, from Proposition 3.1,

$$
\begin{aligned}
\frac{\partial}{\partial \zeta} \mathrm{E}\left[\mathbf{1}_{\left\{N_{T} \geq n_{0}\right\}} f\left(F_{\zeta}\right)\right] & =\mathrm{E}\left[\mathbf{1}_{\left\{N_{T} \geq n_{0}\right\}} f^{\prime}\left(F_{\zeta}\right) \frac{\partial F_{\zeta}}{\partial \zeta}\right] \\
& =\mathrm{E}\left[\mathbf{1}_{\left\{N_{T} \geq n_{0}\right\}} \frac{\partial_{\zeta} F_{\zeta}}{D_{w} F_{\zeta}} D_{w}\left(f\left(F_{\zeta}\right)\right)\right] \\
& =\mathrm{E}\left[f\left(F_{\zeta}\right) D_{w}^{*}\left(\mathbf{1}_{\left\{N_{T} \geq n_{0}\right\}} \frac{\partial_{\zeta} F_{\zeta}}{D_{w} F_{\zeta}}\right)\right] .
\end{aligned}
$$

The extension to the general case is obtained from the bound

$$
\left|\frac{\partial}{\partial \zeta} \mathrm{E}\left[f_{n}\left(F_{\zeta}\right) \mathbf{1}_{\left\{N_{T} \geq n_{0}\right\}}\right]-\mathrm{E}\left[W_{\zeta} f\left(F_{\zeta}\right)\right]\right| \leq\left\|f\left(F_{\zeta}\right)-f_{n}\left(F_{\zeta}\right)\right\|_{L^{p}}\left\|W_{\zeta} \mathbf{1}_{\left\{N_{T} \geq n_{0}\right\}}\right\|_{L^{q}},
$$

and an approximating sequence $\left(f_{n}\right)_{n \in \mathbb{N}}$ of smooth functions.

In the next proposition we focus on a sufficient condition for (4.2) to hold. These conditions can be checked using (2.2).

Proposition 4.2. Assume that $F_{\zeta} \in s_{T}, \zeta \in(a, b)$, and let $1 / q^{\prime}+1 / p^{\prime}=1 / q, p^{\prime}<q^{\prime}$, such that $\partial_{\zeta} F_{\zeta} \in \operatorname{Dom}_{2 q^{\prime}}\left(D_{w}\right), D_{w} F_{\zeta} \in \operatorname{Dom}_{2 q^{\prime}}\left(D_{w}\right)$, and $\left(D_{w} F_{\zeta}\right)^{-1} \in L^{2 q^{\prime}}\left(\left\{N_{T} \geq n_{0}\right\}\right)$. Then (4.2) holds and we have

$$
\frac{\partial}{\partial \zeta} \mathrm{E}\left[f\left(F_{\zeta}\right) \mid N_{T} \geq n_{0}\right]=\mathrm{E}\left[W_{\zeta} f\left(F_{\zeta}\right) \mid N_{T} \geq n_{0}\right], \quad \zeta \in(a, b)
$$


where the weight $W_{\zeta}$ is given by

$$
\begin{aligned}
W_{\zeta}=\frac{\mathbf{1}_{\left\{N_{T} \geq n_{0}\right\}}}{D_{w} F_{\zeta}}\left(\partial_{\zeta} F_{\zeta}\left(\int_{0}^{T} w^{\prime}(t) \mathrm{d} N_{t}-D_{w} \log \left|\partial_{\zeta} F_{\zeta} j_{T, N_{T}}\left(T_{1}, \ldots, T_{N_{T}}\right)\right|+\frac{D_{w} D_{w} F_{\zeta}}{D_{w} F_{\zeta}}\right)\right. \\
\left.\quad-D_{w} \partial_{\zeta} F_{\zeta}\right)
\end{aligned}
$$

and belongs to $L^{q}\left(\Omega, \mathcal{F}_{T}\right)$.

Proof. Since $F_{\zeta} \in s_{T}$ we have, from (3.1),

$$
\begin{aligned}
D_{w}^{*}\left(\mathbf{1}_{\left\{N_{T} \geq n_{0}\right\}} \frac{\partial_{\zeta} F_{\zeta}}{D_{w} F_{\zeta}}\right) & \\
= & \mathbf{1}_{\left\{N_{T} \geq n_{0}\right\}} \frac{\partial_{\zeta} F_{\zeta}}{D_{w} F_{\zeta}}\left(\int_{0}^{T} w^{\prime}(t) \mathrm{d} N_{t}-D_{w} \log j_{T, N_{T}}\left(T_{1}, \ldots, T_{N_{T}}\right)\right) \\
& \quad-D_{w}\left(\mathbf{1}_{\left\{N_{T} \geq n_{0}\right\}} \frac{\partial_{\zeta} F_{\zeta}}{D_{w} F_{\zeta}}\right) \\
= & \frac{\mathbf{1}_{\left\{N_{T} \geq n_{0}\right\}}}{D_{w} F_{\zeta}}\left(\partial_{\zeta} F_{\zeta}\left(\int_{0}^{T} w^{\prime}(t) \mathrm{d} N_{t}-D_{w} \log \left|\partial_{\zeta} F_{\zeta} j_{T, N_{T}}\left(T_{1}, \ldots, T_{N_{T}}\right)\right|+\frac{D_{w} D_{w} F_{\zeta}}{D_{w} F_{\zeta}}\right)\right. \\
& \left.\quad-D_{w} \partial_{\zeta} F_{\zeta}\right) .
\end{aligned}
$$

In order to apply Proposition 4.1 we need to check the domain condition

$$
\mathbf{1}_{\left\{N_{T} \geq n_{0}\right\}} \frac{\partial_{\zeta} F_{\zeta}}{D_{w} F_{\zeta}} \in \operatorname{Dom}_{q}\left(D_{w}^{*}\right)
$$

which is satisfied by Remark 3.1, provided that $W_{\zeta}$, as defined in (4.3), belongs to $L^{q}\left(\Omega, \mathcal{F}_{T}\right)$. By Hölder's inequality we have

$$
\begin{aligned}
\left\|W_{\zeta}\right\|_{L^{q}} \leq & \left\|\left(D_{w} F_{\zeta}\right)^{-1}\right\|_{L^{2 q^{\prime}}\left(\left\{N_{T} \geq n_{0}\right\}\right)}^{2}\left\|\partial_{\zeta} F_{\zeta} D_{w} D_{w} F_{\zeta}\right\|_{L^{q^{\prime}}} \\
+ & \left\|\left(D_{w} F_{\zeta}\right)^{-1}\right\|_{L^{q^{\prime}}\left(\left\{N_{T} \geq n_{0}\right\}\right)} \\
& \quad \times\left\|\partial_{\zeta} F_{\zeta} \int_{0}^{T} w^{\prime}(t) \mathrm{d} N_{t}+\partial_{\zeta} F_{\zeta} D_{w} \log j_{T, N_{T}}\left(T_{1}, \ldots, T_{N_{T}}\right)+D_{w} \partial_{\zeta} F_{\zeta}\right\|_{L^{p^{\prime}}} \\
\leq & \left\|\left(D_{w} F_{\zeta}\right)^{-1}\right\|_{L^{2 q^{\prime}}\left(\left\{N_{T} \geq n_{0}\right\}\right)}\left\|\partial_{\zeta} F_{\zeta}\right\|_{L^{2 q^{\prime}}} \\
& \times\left(\left\|\int_{0}^{T} w^{\prime}(t) \mathrm{d} N_{t}\right\|_{L^{2 p^{\prime}}}\right. \\
& \left.\quad+\left\|D_{w} \log j_{T, N_{T}}\left(T_{1}, \ldots, T_{N_{T}}\right)\right\|_{L^{2 p^{\prime}}}+\left\|D_{w} \partial_{\zeta} F_{\zeta}\right\|_{L^{2 p^{\prime}}}+\left\|D_{w} D_{w} F_{\zeta}\right\|_{L^{2 q^{\prime}}}\right),
\end{aligned}
$$

which, together with Lemma 3.1, completes the proof.

In the case of a Poisson process with deterministic intensity $\lambda \in \mathcal{C}^{1}\left(\mathbb{R}_{+}\right)$we have

$$
W_{\zeta}=\mathbf{1}_{\left\{N_{T} \geq n_{0}\right\}}\left(\frac{\partial_{\zeta} F_{\zeta}}{D_{w} F_{\zeta}}\left(\int_{0}^{T} w^{\prime}(t) \mathrm{d} N_{t}-\int_{0}^{T} w(t) \frac{\lambda^{\prime}(t)}{\lambda(t)} \mathrm{d} N_{t}+\frac{D_{w} D_{w} F_{\zeta}}{D_{w} F_{\zeta}}\right)-\frac{D_{w} \partial_{\zeta} F_{\zeta}}{D_{w} F_{\zeta}}\right) .
$$


In general, we assume that the Janossy densities $j_{T, n}$ are known in order to compute the weight $W_{\zeta}$, while the density of $F$ may not be analytically computable or may be unknown as in the following example.

Now consider a compound point process of the form

$$
X_{t}=\sum_{k=1}^{N_{t}} Y_{k}, \quad t \in \mathbb{R}_{+}
$$

where $\left(Y_{k}\right)_{k \geq 1}$ is a sequence of random marks independent of $\left(N_{t}\right)_{t \in \mathbb{R}_{+}}$, such that there exists $c_{2}>0$ such that $Y_{k} \geq c_{2}>0$ a.s., $k \geq 1$. We make the additional assumption that

$$
j_{T, n}\left(t_{1}, \ldots, t_{n}\right) \leq c_{0}^{n}, \quad t_{1}, \ldots, t_{n} \in[0, T]^{n},
$$

$k=1, \ldots, n, n \geq 1$.

Proposition 4.3. Consider two $\mathcal{C}^{1}$ functions $g:(a, b) \rightarrow \mathbb{R}$ and $h:[a, b] \times[0, T] \rightarrow \mathbb{R}$ such that $\partial h / \partial t$ does not vanish on $[a, b] \times[0, T]$, and let

$$
F_{\zeta}=g(\zeta)+\int_{0}^{T} h(\zeta, t) \mathrm{d} X_{t}=g(\zeta)+\sum_{k=1}^{N_{t}} Y_{k} h\left(\zeta, T_{k}\right), \quad \zeta \in(a, b)
$$

Let $\alpha>0$ and

$$
w(t)=t^{\alpha}(T-t)^{\alpha}, \quad t \in[0, T] .
$$

Then (4.2) holds whenever $n_{0} \geq 2 \alpha$ and we have

$$
\frac{\partial}{\partial \zeta} \mathrm{E}\left[f\left(F_{\zeta}\right) \mid N_{T} \geq n_{0}\right]=\mathrm{E}\left[W_{\zeta} f\left(F_{\zeta}\right) \mid N_{T} \geq n_{0}\right], \quad \zeta \in(a, b)
$$

where the weight $W_{\zeta}$ belongs to $L^{q}(\Omega), \zeta \in(a, b)$.

Proof. We have

$$
\partial_{\zeta} F_{\zeta}=g^{\prime}(\zeta)+\int_{0}^{T} \frac{\partial h}{\partial \zeta}(\zeta, t) \mathrm{d} X_{t}
$$

which belongs to $L^{p}(\Omega)$ for all $p \geq 1$. Since the gradient $D_{w}$ does not act on $Y_{k}, k \in \mathbb{N}$, these random variables can be considered as constants in the integration by parts formula (3.2) and we have

$$
D_{w} F_{\zeta}=-\int_{0}^{T} w(t) \frac{\partial h}{\partial t}(\zeta, t) \mathrm{d} X_{t} .
$$

Moreover, there exists a $c_{1}>0$ such that

$$
\left|\frac{\partial h}{\partial t}(\zeta, t)\right| \geq c_{1}>0, \quad(\zeta, t) \in[a, b] \times[0, T] ;
$$


hence, for any $p^{\prime}, q^{\prime}$ such that $1 / q^{\prime}+1 / p^{\prime}=1 / q$, we have

$$
\begin{aligned}
& \left\|\left(D_{w} F_{\zeta}\right)^{-1}\right\|_{L^{2 q^{\prime}}\left(\left\{N_{T} \geq n_{0}\right\}\right)}^{2 q^{\prime}} \\
& =\mathrm{E}\left[\mathbf{1}_{\left\{N_{T} \geq n_{0}\right\}}\left|\int_{0}^{T} w(t) \frac{\partial h}{\partial t}(\zeta, t) \mathrm{d} X_{t}\right|^{-2 q^{\prime}}\right] \\
& =\mathrm{E}\left[\sum_{n=n_{0}}^{\infty} \frac{1}{n !} \int_{0}^{T} \cdots \int_{0}^{T} \frac{j_{T, n}\left(t_{1}, \ldots, t_{n}\right)}{\left|\sum_{k=1}^{n} Y_{k} t_{k}^{\alpha}\left(T-t_{k}\right)^{\alpha}\left(\partial h\left(\zeta, t_{k}\right) / \partial t\right)\right|^{2 q^{\prime}}} \mathrm{d} t_{1} \cdots \mathrm{d} t_{n}\right] \\
& \leq \frac{2^{2 \alpha q^{\prime}}}{\left(c_{1} c_{2}\right)^{2 q^{\prime}}} \sum_{n=n_{0}}^{\infty} \frac{2^{n} c_{0}^{n}}{n !} \int_{0}^{1 / 2} \cdots \int_{0}^{1 / 2}\left(\sum_{k=1}^{n} t_{k}^{\alpha}\right)^{-2 q^{\prime}} \mathrm{d} t_{1} \cdots \mathrm{d} t_{n} \\
& \leq \frac{2^{2 \alpha q^{\prime}}}{\left(c_{1} c_{2}\right)^{2 q^{\prime}}} \sum_{n=n_{0}}^{\infty} \frac{c_{0}^{n}}{n !}\left(\frac{1}{4^{\alpha q^{\prime}}}+2^{n} \int_{\left\{\sum_{k=1}^{n} t_{k}^{2} \leq 1 / 4\right\}}\left(\sum_{k=1}^{n} t_{k}^{2}\right)^{-\alpha q^{\prime}} \mathrm{d} t_{1} \cdots \mathrm{d} t_{n}\right) \\
& =\frac{2^{2 \alpha q^{\prime}}}{\left(c_{1} c_{2}\right)^{2 q^{\prime}}} \sum_{n=n_{0}}^{\infty} \frac{c_{0}^{n}}{n !}\left(\frac{1}{4^{\alpha q^{\prime}}}+\frac{2^{n+1} \pi^{n / 2}}{\Gamma(n / 2)} \int_{0}^{1 / 2} r^{n-1-2 \alpha q^{\prime}} \mathrm{d} r\right) \\
& =\left(c_{1} c_{2}\right)^{-2 q^{\prime}} \sum_{n=n_{0}}^{\infty} \frac{c_{0}^{n}}{n !}\left(1+\frac{2^{1+4 \alpha q^{\prime}} \pi^{n / 2}}{\Gamma(n / 2)\left(n-2 \alpha q^{\prime}\right)}\right) \text {, }
\end{aligned}
$$

which is finite whenever $n_{0}>2 \alpha q^{\prime}>2 \alpha$; hence, we can apply Proposition 4.2.

In practice we can choose $n_{0}=1$ provided that $\alpha \in\left(0, \frac{1}{2}\right)$. Note that at least four jumps can be required in other situations; see e.g. Proposition 3.2 of [1] in the Poisson case.

For example, taking $h(\zeta, t)=\mathrm{e}^{-\zeta t}$, the weight $W_{\zeta}$ corresponding to the sensitivity

$$
\frac{\partial}{\partial \zeta} \mathrm{E}\left[\mathbf{1}_{\left\{N_{T} \geq n_{0}\right\}} f\left(F_{\zeta}\right)\right]=\mathrm{E}\left[\mathbf{1}_{\left\{N_{T} \geq n_{0}\right\}} W_{\zeta} f\left(F_{\zeta}\right)\right]
$$

with respect to the parameter $\zeta>0$ is given on $\left\{N_{T} \geq n_{0}\right\}$ by

$$
\begin{aligned}
W_{\zeta}= & -\frac{1}{\zeta}+\frac{\int_{0}^{T} w(t) t \mathrm{e}^{-\zeta t} \mathrm{~d} X_{t}}{\int_{0}^{T} w(t) \mathrm{e}^{-\zeta t} \mathrm{~d} X_{t}} \\
& -\frac{\int_{0}^{T} t \mathrm{e}^{-\zeta t} \mathrm{~d} X_{t}}{\zeta \int_{0}^{T} w(t) \mathrm{e}^{-\zeta t} \mathrm{~d} X_{t}}\left(\frac{\int_{0}^{T} w(t)\left(\zeta w(t)-w^{\prime}(t)\right) \mathrm{e}^{-\zeta t} \mathrm{~d} X_{t}}{\int_{0}^{T} w(t) \mathrm{e}^{-\zeta t} \mathrm{~d} X_{t}}\right. \\
& \left.\quad-\int_{0}^{T} w^{\prime}(t) \mathrm{d} N_{t}+D_{w} \log j_{T, N_{T}}\left(T_{1}, \ldots, T_{N_{T}}\right)\right) .
\end{aligned}
$$

\section{Density estimation}

In this section we apply the above results to the computation of the conditional density $\phi_{F}\left(\cdot \mid N_{T} \geq n_{0}\right)$ of a random variable $F$ with respect to the Lebesgue measure, written as the derivative

$$
\phi_{F}\left(y \mid N_{T} \geq n_{0}\right)=-\frac{\mathrm{d}}{\mathrm{d} y} \mathrm{E}\left[f(F-y) \mid N_{T} \geq n_{0}\right], \quad y \in \mathbb{R},
$$

with $f=\mathbf{1}_{(0, \infty)}$, i.e. we take $F^{\zeta}=F-\zeta, \zeta \in \mathbb{R}$. 


\subsection{Kernel estimators}

The standard kernel estimator of the density $\phi_{F}$ with respect to the Lebesgue measure is given by

$$
\phi_{F}(y) \simeq \frac{1}{h} \mathrm{E}\left[K\left(\frac{F-y}{h}\right)\right] \simeq \frac{1}{N h} \sum_{k=1}^{N} K\left(\frac{F(k)-y}{h}\right),
$$

where $K$ is a continuous positive function such that

$$
\int_{-\infty}^{\infty} K(x) \mathrm{d} x=1
$$

\subsection{Malliavin estimators}

Taking $F_{y}=F-y$, Proposition 4.2 yields the following corollary.

Corollary 5.1. Assume that $F \in s_{T}$ and let $1 / q^{\prime}+1 / p^{\prime}=1 / q, p^{\prime}<q^{\prime}$, such that $D_{w} F \in$ $\operatorname{Dom}_{2 q^{\prime}}\left(D_{w}\right)$ and $\left(D_{w} F\right)^{-1} \in L^{2 q^{\prime}}\left(\left\{N_{T} \geq n_{0}\right\}\right)$. Then we have

$$
\frac{\partial}{\partial y} \mathrm{E}\left[\mathbf{1}_{\left\{N_{T} \geq n_{0}\right\}} f(F-y)\right]=\mathrm{E}\left[W \mathbf{1}_{\left\{N_{T} \geq n_{0}\right\}} f(F-y)\right]
$$

for $f$ bounded and measurable on $\mathbb{R}$, where

$$
W=\frac{\mathbf{1}_{\left\{N_{T} \geq n_{0}\right\}}}{D_{w} F}\left(\int_{0}^{T} w^{\prime}(t) \mathrm{d} N_{t}-D_{w} \log j_{T, N_{T}}\left(T_{1}, \ldots, T_{N_{T}}\right)+\frac{D_{w} D_{w} F}{D_{w} F}\right)
$$

belongs to $L^{q}(\Omega)$.

In particular, taking $f=-\mathbf{1}_{[0, \infty)}$ we obtain

$\phi_{F}\left(y \mid N_{T} \geq n_{0}\right)=-\frac{\mathrm{d}}{\mathrm{d} y} \mathrm{E}\left[\mathbf{1}_{[0, \infty)}(F-y) \mid N_{T} \geq n_{0}\right]=-\mathrm{E}\left[W \mathbf{1}_{[0, \infty)}(F-y) \mid N_{T} \geq n_{0}\right]$,

$y \in \mathbb{R}$, where the weight $W$ is independent of $y$ and of any bandwidth parameter. Here the condition $\{F>y\}$ in (5.3) with $y>0$ actually ensures the integrability of $W \mathbf{1}_{[0, \infty)}(F-y)$ on $\left\{N_{T} \geq 1\right\}$. This yields the estimate

$$
\phi_{F}\left(y \mid N_{T} \geq n_{0}\right) \simeq-\frac{\mathbf{1}_{\left\{N_{T} \geq n_{0}\right\}}}{N \mathrm{P}\left(N_{T} \geq n_{0}\right)} \sum_{i=1}^{N} W(i) \mathbf{1}_{[0, \infty)}(F(i)-y) .
$$

For the case in which $F=\int_{0}^{T} h(t) \mathrm{d} X_{t}$, the relation

$$
D_{w} D_{w} F=\int_{0}^{T} w(t)\left(\frac{\partial h}{\partial t}(\zeta, t) w^{\prime}(t)+\frac{\partial^{2} h}{\partial t^{2}}(\zeta, t) w(t)\right) \mathrm{d} X_{t}
$$

yields

$$
\begin{aligned}
W= & \frac{\mathbf{1}_{\left\{N_{T} \geq n_{0}\right\}}}{\int_{0}^{T} w(t)(\partial h(\zeta, t) / \partial t) \mathrm{d} X_{t}} \\
& \times\left(\int_{0}^{T} w^{\prime}(t) \mathrm{d} N_{t}-D_{w} \log j_{T, N_{T}}\left(T_{1}, \ldots, T_{N_{T}}\right)\right. \\
& \left.\quad-\frac{\int_{0}^{T} w(t)\left((\partial h(\zeta, t) / \partial t) w^{\prime}(t)+\left(\partial^{2} h(\zeta, t) / \partial t^{2}\right) w(t)\right) \mathrm{d} X_{t}}{\int_{0}^{T} w(t)(\partial h(\zeta, t) / \partial t) \mathrm{d} X_{t}}\right) .
\end{aligned}
$$




\subsection{Modified kernel estimators}

When $D_{w} F$ is close to 0 , the value of $W$ becomes large, due to the division by $D_{w} F$ in (5.4); hence, when $y$ is small the term $W \mathbf{1}_{[0, \infty)}(F-y)$ is allowed to be nonzero for small values of $F$, and it has a large variance. A variance reduction technique called localization has been introduced by Fournié et al. [10] to deal with related problems on the Wiener space. Here we apply a similar procedure to construct a modified kernel estimator using Malliavin weights. For this we will consider a decomposition of the form

$$
\mathbf{1}_{[0, \infty)}=f+g,
$$

where $g$ is a $\mathcal{C}^{1}$ function. In the following proposition we obtain an analog of Theorem 2.1 of [12], via a somewhat simpler argument, under the hypotheses of Proposition 4.1.

Proposition 5.1. Assume that $F \in s_{T}$ and let $1 / q^{\prime}+1 / p^{\prime}=1 / q, p^{\prime}<q^{\prime}$, such that $D_{w} F \in \operatorname{Dom}_{2 q^{\prime}}\left(D_{w}\right)$ and $\left(D_{w} F\right)^{-1} \in L^{2 q^{\prime}}\left(\left\{N_{T} \geq n_{0}\right\}\right)$, and let $f$ be a function on $\mathbb{R}$ such that $f(0)=1, f(x)=0, x<0$, and $\mathbf{1}_{(0, \infty)} f^{\prime} \in \overline{L^{2}}((0, \infty))$. We have, for all $\eta>0$,

$$
\begin{aligned}
& \phi_{F}\left(y \mid N_{T} \geq n_{0}\right) \\
& \quad=-\mathrm{E}\left[W f\left(\frac{F-y}{\eta}\right) \mid N_{T} \geq n_{0}\right]-\frac{1}{\eta} \mathrm{E}\left[\mathbf{1}_{\{F>y\}} f^{\prime}\left(\frac{F-y}{\eta}\right) \mid N_{T} \geq n_{0}\right], \quad y \in \mathbb{R},
\end{aligned}
$$

where $W$ is given by (5.2).

Proof. Letting $g=\mathbf{1}_{[0, \infty)}-f$ we have

$$
\begin{aligned}
\phi_{F}\left(y \mid N_{T} \geq n_{0}\right)= & -\frac{\mathrm{d}}{\mathrm{d} y} \mathrm{E}\left[\mathbf{1}_{[0, \infty)}(F-y) \mid N_{T} \geq n_{0}\right] \\
= & -\frac{\mathrm{d}}{\mathrm{d} y} \mathrm{E}\left[f\left(\frac{F-y}{\eta}\right) \mid N_{T} \geq n_{0}\right]-\frac{\mathrm{d}}{\mathrm{d} y} \mathrm{E}\left[g\left(\frac{F-y}{\eta}\right) \mid N_{T} \geq n_{0}\right] \\
= & -\mathrm{E}\left[W f\left(\frac{F-y}{\eta}\right) \mid N_{T} \geq n_{0}\right] \\
& -\frac{1}{\eta} \mathrm{E}\left[\mathbf{1}_{\{F>y\}} f^{\prime}\left(\frac{F-y}{\eta}\right) \mid N_{T} \geq n_{0}\right], \quad y \in \mathbb{R},
\end{aligned}
$$

where $W$ is given by (5.4).

Letting $K(x)=-\mathbf{1}_{(0, \infty)}(x) f^{\prime}(x)$, this leads, by Monte Carlo approximation, to a family of corrected kernel estimators

$$
\phi_{F}\left(y \mid N_{T} \geq n_{0}\right) \simeq \frac{\mathbf{1}_{\left\{N_{T} \geq n_{0}\right\}}}{N \mathrm{P}\left(N_{T} \geq n_{0}\right)} \sum_{i=1}^{N}\left(\frac{1}{\eta} K\left(\frac{F(i)-y}{\eta}\right)-W(i) f\left(\frac{F(i)-y}{\eta}\right)\right),
$$

depending on $\eta>0$. Note that (5.5) is an equality, whereas the standard kernel estimate

$$
\phi_{F}\left(y \mid N_{T} \geq n_{0}\right) \simeq \frac{1}{\eta} \mathrm{E}\left[K\left(\frac{F-y}{\eta}\right) \mid N_{T} \geq n_{0}\right], \quad y \in \mathbb{R},
$$

is only an approximation. 
The method for the determination of an optimal kernel $f: \mathbb{R} \rightarrow \mathbb{R}$ and a bandwidth parameter $\eta>0$ by minimization of

$$
\mathrm{E}\left[\mathbf{1}_{\left\{N_{T} \geq n_{0}\right\} \cap\{F>y\}}\left(W f\left(\frac{F-y}{\eta}\right)-\frac{1}{\eta} \mathbf{1}_{\{F>y\}} f^{\prime}\left(\frac{F-y}{\eta}\right)\right)^{2}\right], \quad y \in \mathbb{R},
$$

as in [12, p. 446], also applies here and yields

$$
f(x)=\mathbf{1}_{[0, \infty)}(x) \mathrm{e}^{-\lambda x}, \quad x \in \mathbb{R},
$$

and $\eta_{\mathrm{opt}}=\|W\|_{L^{2}\left(\left\{N_{T} \geq n_{0}\right\}\right)}^{-1}$, for any $\lambda>0$. Note that the criterion of optimality for $\eta$ is not linked to the number of samples $N$, as is the case for the optimal decrease in $N^{-1 / 4}$ of the kernel estimator bandwidth parameter $h$.

\section{Numerical results}

Our results are illustrated by Monte Carlo density estimations with 10000 samples for the random variable

$$
F_{r}:=\alpha(r) \int_{0}^{T} \mathrm{e}^{-r t} \mathrm{~d} N_{t},
$$

where $\left(N_{t}\right)_{t \in \mathbb{R}_{+}}$is a log-normal renewal process and $T=5, \sigma=0.3$, and $\alpha(r)=\exp ((1+$ $\left.r)^{2}-1\right)$ is a parameter chosen to enhance the readability of the simulation graphs. Clearly the law of $F_{r}$ has a Dirac mass at $y=0$, and we are interested in the values of the density on $\mathbb{R} \backslash\{0\}$ with respect to the Lebesgue measure.

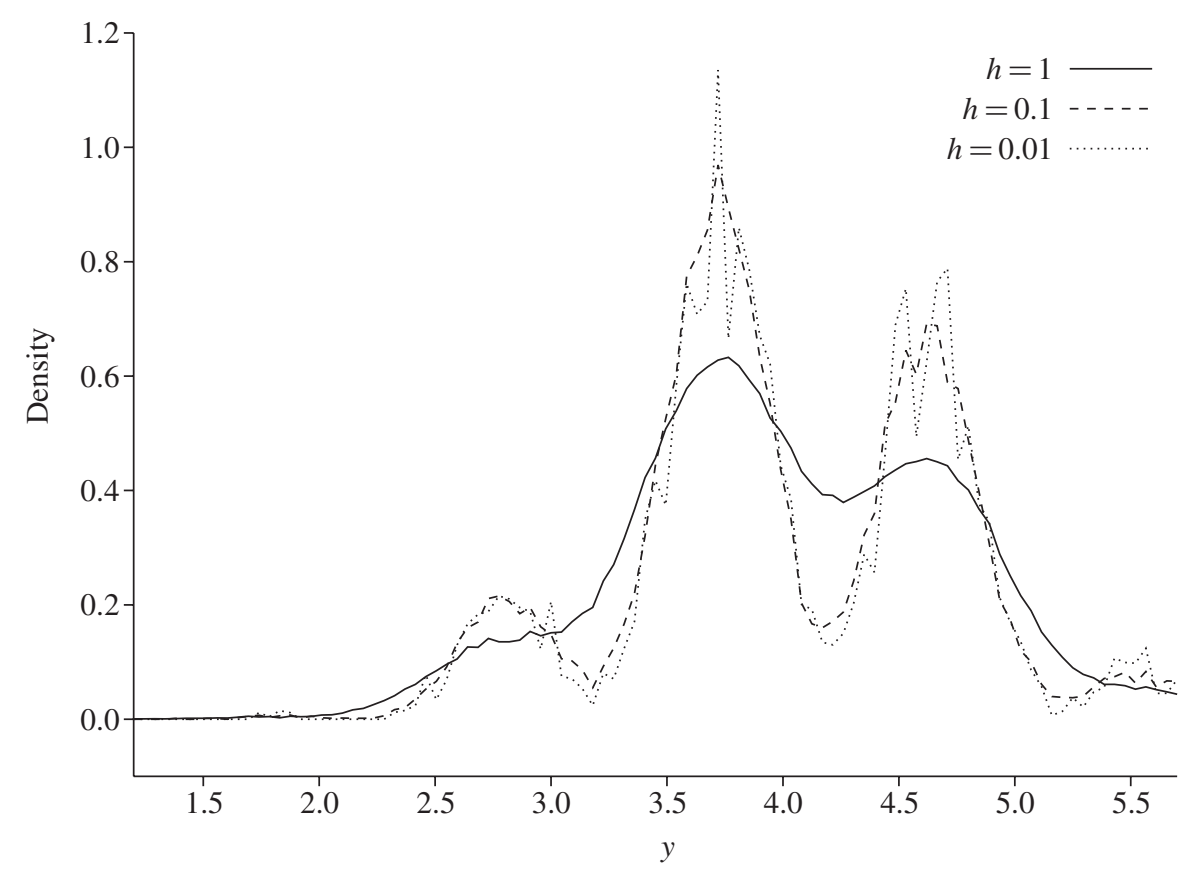

FIGURE 1: Kernel estimations of $\phi_{F_{r}}$ with 10000 samples and $r=0.2$. 


\subsection{Kernel estimators}

We start by comparing several kernel estimators in Figure 1, with

$$
K(x)=\frac{\pi}{2} \mathbf{1}_{[-1 / 2,1 / 2]}(x) \cos (\pi x)
$$

and $\eta=1,0.1,0.01$.

\subsection{Malliavin estimator}

For the Malliavin method we use (5.3), where the weight $W$ given by

$$
\begin{gathered}
W=-\frac{\mathbf{1}_{\left\{N_{T} \geq n_{0}\right\}}}{r \alpha(r) \int_{0}^{T} w(t) \mathrm{e}^{-r t} \mathrm{~d} N_{t}}\left(\int_{0}^{T} w^{\prime}(t) \mathrm{d} N_{t}-D_{w} \log j_{T, N_{T}}\left(T_{1}, \ldots, T_{N_{T}}\right)\right. \\
\left.+\frac{\int_{0}^{T} w(t)\left(r w(t)-w^{\prime}(t)\right) \mathrm{e}^{-r t} \mathrm{~d} N_{t}}{\int_{0}^{T} w(t) \mathrm{e}^{-r t} \mathrm{~d} N_{t}}\right)
\end{gathered}
$$

is independent of $y$ and of any bandwidth parameter. The result of this estimation is shown in Figure 2 .

The graph labeled 'exact value' has been obtained using finite differences with $10^{7}$ samples. We can check, in Figure 2, that although the Malliavin estimator, (5.3), yields more precise values than the kernel estimator, (5.1), when $y$ is large, it behaves badly for small values of $y$ due to a higher variance of $W \mathbf{1}_{[0, \infty)}(F-y)$ in this situation. This phenomenon is dealt with by the modified kernel estimator introduced in Section 5 by localization.

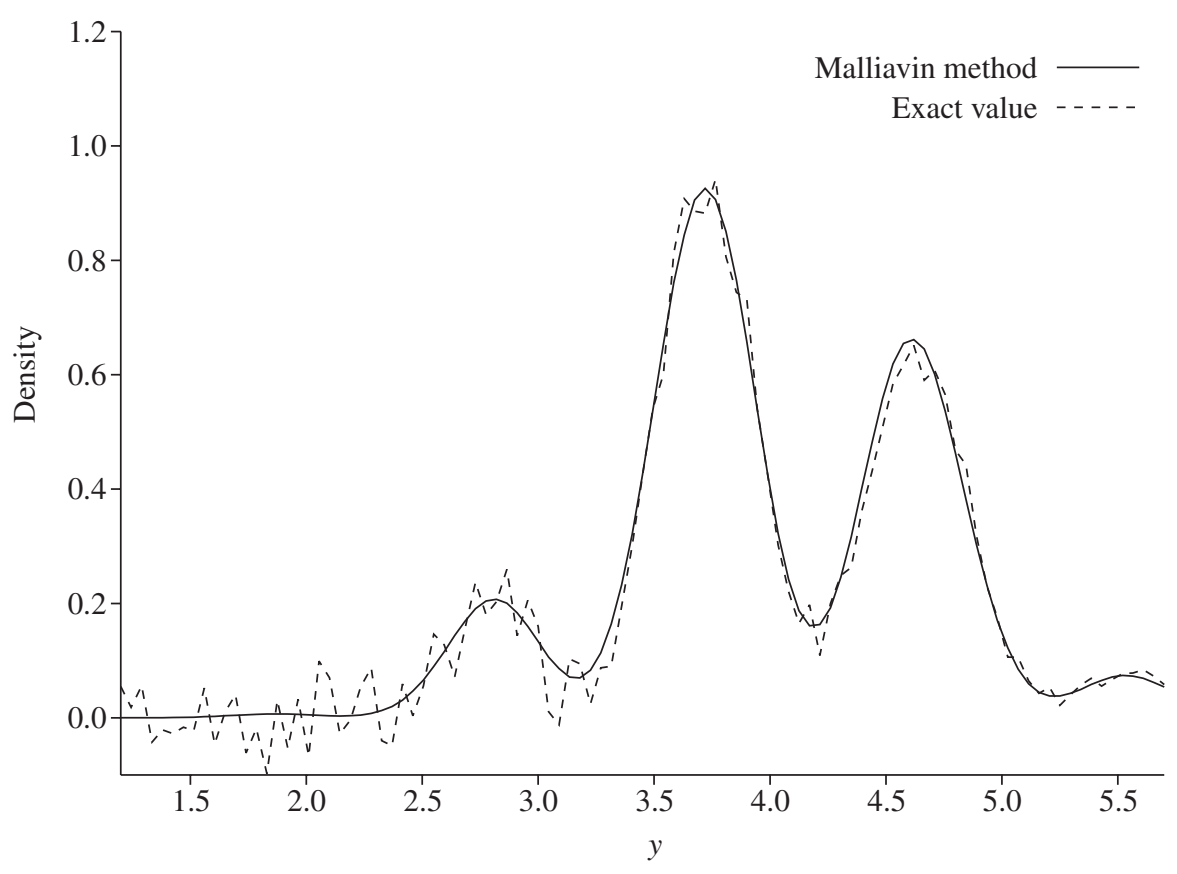

FIGURE 2: The probability density of $F_{r}$ for $r=0.2$ (Malliavin method with 10000 samples). 


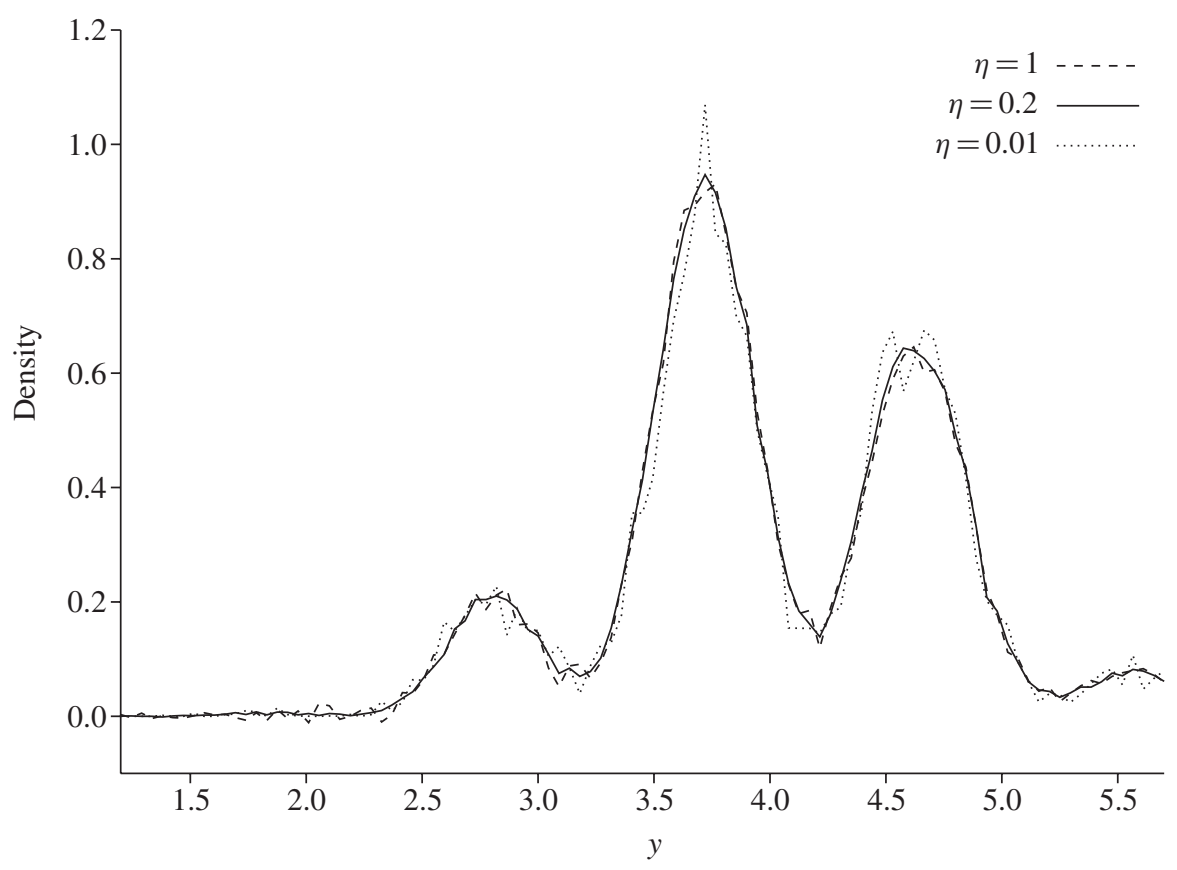

FIGURE 3: Modified kernel estimates of $\phi_{F_{r}}$ with 10000 samples and $r=0.2$.

\subsection{Modified kernel estimators}

Figure 3 shows the result of the modified kernel estimation for $\eta=1,0.2,0.01$, for comparison with the standard kernel estimate of Figure 1. The modified kernel estimator does depend on a parameter called $\eta$, but it appears more stable and less sensitive to variations of $\eta$ than standard kernel estimators are sensitive to the value of the bandwidth parameter $h$. In our setting we found $\eta_{\text {opt }}=0.1963$ by Monte Carlo simulation and we used the optimal kernel $K(x)=\mathbf{1}_{(0, \infty)}(x) \mathrm{e}^{-x}$.

\section{Conclusion}

Both Malliavin and modified kernel estimators are consistent. The performances of kernel estimators are dependent on the choice of a bandwidth parameter $\eta$. The results of the Malliavin method are independent of $\eta$ but may be degraded as the weight variance increases. In the examples considered in this paper, the latter performs better than the other estimators.

\section{Acknowledgement}

We thank Gérard Grancher (LMRS Rouen) for pointing out to us the connection between kernel estimators and a preliminary version of this paper.

\section{References}

[1] Bally, V., Bavouzet-Morel, M.-P. and Messaoud, M. (2007). Integration by parts formula for locally smooth laws and applications to sensitivity computations. Ann. Appl. Prob. 17, 33-66.

[2] Bavouzet-Morel, M.-P. And Messaoud, M. (2006). Computation of Greeks using Malliavin's calculus in jump type market models. Electron. J. Prob. 11, 276-300. 
[3] Carlen, E. and Pardoux, E. (1990). Differential calculus and integration by parts on Poisson space. In Stochastics, Algebra and Analysis in Classical and Quantum Dynamics (Marseille, 1988; Math. Appl. 59), Kluwer, Dordrecht, pp. 63-73.

[4] Daley, D. J. And Vere-Jones, D. (2003). An Introduction to the Theory of Point Processes, Vol. I, Probability and Its Applications. Springer, New York.

[5] Dash, J. W. (2004). Quantitative Finance and Risk Management. World Scientific, River Edge, NJ.

[6] Davis, M. H. A. and Johansson, M. P. (2006). Malliavin Monte Carlo Greeks for jump diffusions. Stoch. Process. Appl. 116, 101-129.

[7] Debelley, V. and Privault, N. (2004). Sensitivity analysis of European options in jump diffusion models via the Malliavin calculus on Wiener space. Preprint, Université de La Rochelle.

[8] El Khatib, Y. and Privault, N. (2004). Computations of Greeks in markets with jumps via the Malliavin calculus. Finance Stoch. 4, 161-179.

[9] Elliott, R. J. And Tsoi, A. H. (1993). Integration by parts for Poisson processes. J. Multivariate Anal. 44, 179-190.

[10] Fournié, E., Lasry, J. M., Lebuchoux, J. And Lions, P. L. (2001). Applications of Malliavin calculus to Monte-Carlo methods in finance. II. Finance Stoch. 5, 201-236.

[11] Fournié, E. et al. (1999). Applications of Malliavin calculus to Monte Carlo methods in finance. Finance Stoch. 3, 391-412.

[12] Kohatsu-Higa, A. And Pettersson, R. (2002). Variance reduction methods for simulation of densities on Wiener space. SIAM J. Numer. Anal. 40, 431-450.

[13] Nualart, D. (1995). The Malliavin Calculus and Related Topics. Springer, New York.

[14] Parzen, E. (1962). On estimation of a probability density function and mode. Ann. Math. Statist. 33, 1065-1076.

[15] Privault, N. (1994). Chaotic and variational calculus in discrete and continuous time for the Poisson process. Stoch. Stoch. Reports 51, 83-109.

[16] Privault, N. AND WeI, X. (2004). A Malliavin calculus approach to sensitivity analysis in insurance. Insurance Math. Econom. 35, 679-690.

[17] Rosenblatt, M. (1956). Remarks on some nonparametric estimates of a density function. Ann. Math. Statist. 27, 832-837.

[18] Srinivasan, S. K. (1969). Stochastic Theory and Cascade Processes. American Elsevier, New York. 